\title{
Interfacing the Skin
}

\section{FRANK MELENDEZ}

City College of New York
NANCY DINIZ

Rensselaer Polytechnic Institute
Several technologies are converging to drastically change local and global spatiotemporal relationships, including autonomous robotics, cyber-physical systems, ubiquitous sensing networks, and synthetic biological systems. These technologies provide architects and designers with opportunities to redefine models of human-machine-environment interactions that encompass more complex methods of simulated intelligence and nuanced response across a range of scales from the micro to the macro.

This poster presents architectural design research into machinic instruments that emerge as morphological responses to biotic and abiotic phenomena at the interface of bodies and ecological systems across a variety of scales. This includes the design and production of a series of small scale wearable devices that operate as liminal mechanisms, creating a dynamic boundary between the body and the environment through the use of biometrics and environmental data. This conceptual framework for architecture as an extension of the body is achieved through the implementation of computational tools, sensing technologies, and biofabrication processes. 


\section{INTERFACING THE SKIN}

\section{Biometrics and Biofabrication in Architectural Design}

Several technologies are converging to drastically change local and global spatiotemporal relationships, including autonomous robotics, cyber-physical systems, ubiquitous sensing networks, and synthetic biological systems. These technologies provide architects and designers with opportunities to redefine models of human-machine-environment interactions that encompass more complex methods of simulated intelligence and nuanced response across a range of scales from the micro to the macro. This poster presents architectural design research into machinic instruments that emerge as morphological responses to cross a variety of scales. small scale wearable devices that operate as liminal mechanisms, creating a dynamic boundary between the body and the environment through the use of biometrics and environmental data. This conceptual framework for architecture as an extension of the body is achieved through the implementation of computational tools, sensing technologies, and biofabrication processes.
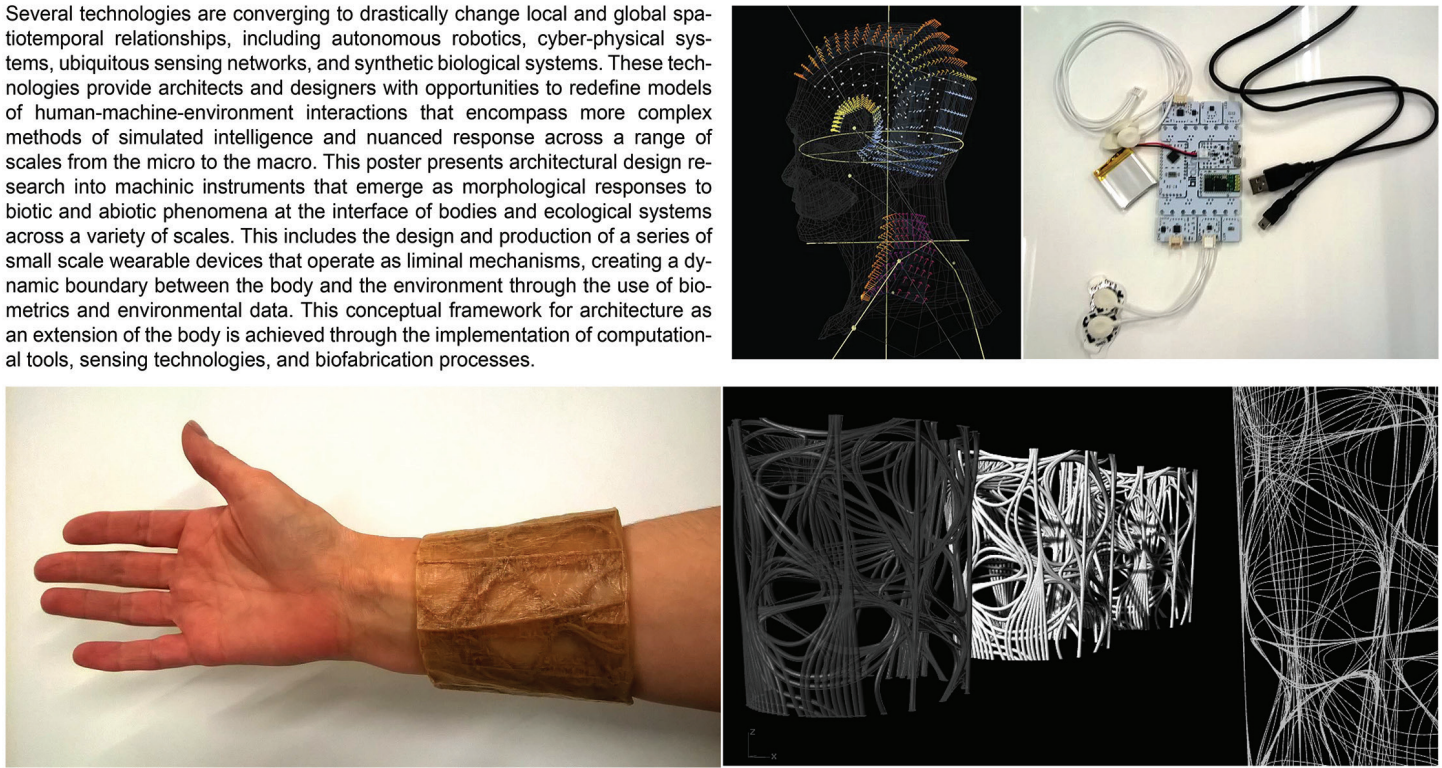

SENSING TECHNOLOGIES alter our ability to imagine constructed systems in highly nuanced relationships between internal bodily signals and surrounding atmospheric data, requiring an expanded view of networked and object oriented relationships between bodies, designed devices, and regional and global environments. This project explores the potential for architectures that utilize and integrate biometric data (heart rate, electrodermal activity) and atmospheric flows (temperature, light) in determining body-machine-environment relationships.

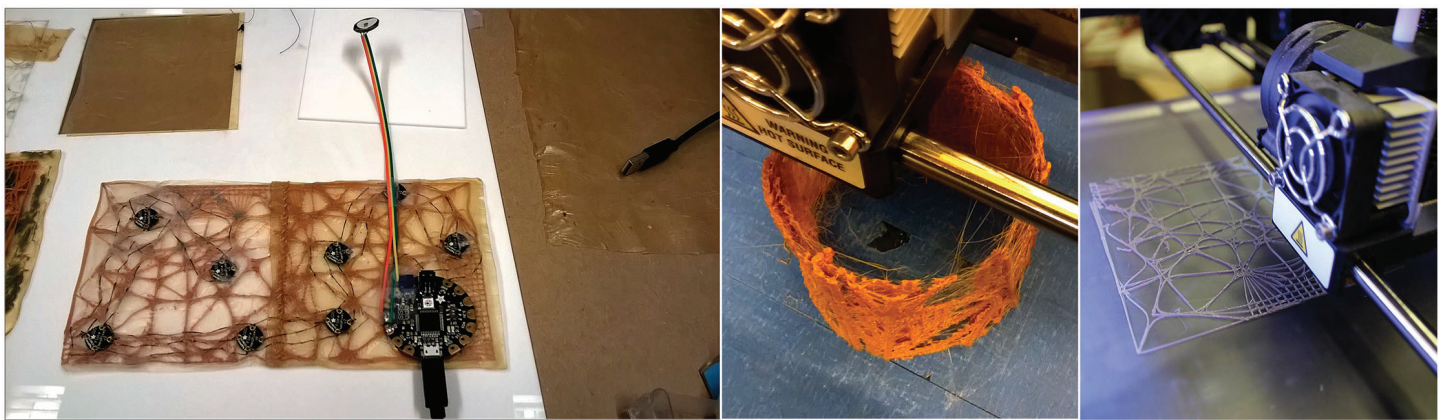

PHYSICAL COMPUTING and computational tools have significantly altered traditional methods of architectural design by enabling an evolved means of measuring, understanding, and organizing data from complex systems and networks. These platforms support the design of interactive systems that sense and respond to fluctuating biological and environmental conditions.

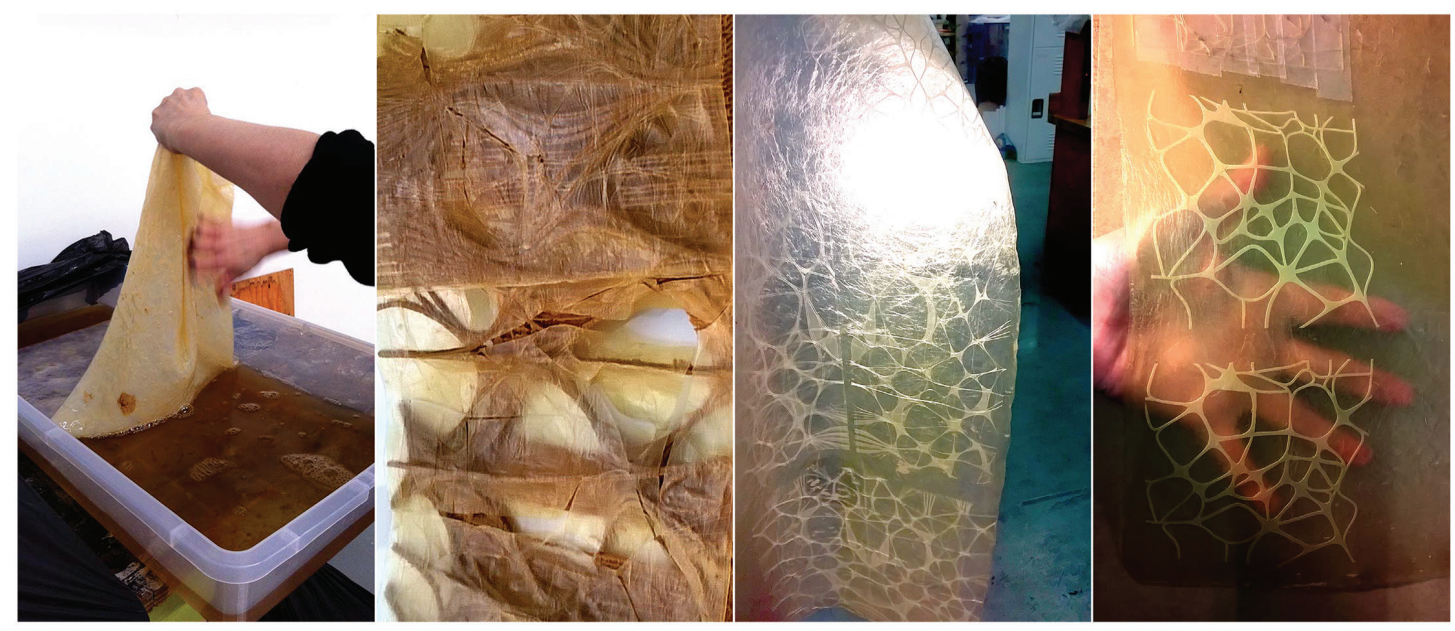

BIOFABRICATION PROCESSES This research utilizes bacterial cellulose, as a means of growing biomaterials for architectural membranes. The ingredients necessary for biofabricating bacterial cellulose, are available globally, however, regional and micro climatic conditions affect nuances in the growing process through the use of local resources available in the region. This provides opportunities to calibrate ecological systems that reduce the waste of local resources. 